\title{
Claims of hidden agenda on French waste
}

[PARIS] The French government has announced plans to create a deep storage centre and two underground laboratories for studying the disposal of nuclear waste in clay and granite localities.

But critics say the plans reveal the government's long-term intentions on the disposal of nuclear waste, and as such conflict with a resolution to keep such decisions open.

The plans, revealed last week, are among a collection of measures relating to the French nuclear industry, including an attempt to improve the transparency of the industry and the organization of its security.

The laboratory to investigate clay sites will be set up at Bure in Meuse, eastern France. According to the National Agency for the Management of Radioactive Waste (ANDRA), it will begin operation in 2003.

The location of the second laboratory, on a granite site, has not yet been chosen. La Chapelle-Bâton in Vienne, western France, near Poitiers, has been suggested by ANDRA, but is no longer being considered.
Preliminary studies showed that the geological characteristics of the site will probably prevent it being used for waste disposal.

The underground repository, which will be less than 20 metres deep, is likely to be set up in Gard in the south of France, after the site has been "scientifically verified".

A report from the Commission on Atomic Energy has indicated that this type of storage is possible. But it "should be accompanied by a programme of research, in particular on methods of cooling".

The Green party, who are members of France's coalition government, sees the decision to drop La Chapelle-Bâton as an indication that the two laboratories will be not only the location of preliminary studies but also the site of future waste-disposal facilities.

The Greens have accused the government of giving in to the nuclear lobby on this matter. But the government insists that it plans to stick to the 'loi Bataille' of 1991, and the concept of 'reversibility', maintaining the chance of keeping access to the stored waste.
The 1991 law specifies that all options for storing nuclear waste should be investigated to ensure that parliament is in full possession of the facts about alternatives when it chooses the method of storage in 2006 (see Nature 390, 322; 1997).

A report of the National Assessment Committee, an independent body, has established a clear link between the type of waste and the type of storage. In particular, the report specifies that "deep storage, involving the triple barrier of a container, a constructed barrier, and then geological barriers, makes reversibility very difficult, if not improbable in the long term". It also argues that "reversibility is easy in subsurface repositories".

Despite this, ecologists are concerned that both the committee and the government are speaking about "definite storage" for the so-called 'B wastes' with medium radioactivity and long lifetimes, without specifying precisely what this means, but contrasting it to subsurface storage.

Eric Glover

\section{Search hopes to detect laser signals from extraterrestrial life}

[воSTON] Researchers at Harvard University and the University of California at Berkeley have begun combing the heavens for flashes of laser light emitted by alien civilizations.

In October, the Harvard group, led by physicist Paul Horowitz, mounted a camera on a 61-inch optical telescope at the Oak Ridge Observatory in Harvard. The device rides 'piggyback' on a survey of 2,500 Sunlike stars, part of a search for extrasolar planets run by the Harvard-Smithsonian Center for Astrophysics, and uses about a third of the light from the telescope in its search for short laser pulses. They have found 30 intriguing signals from the nearly 1,000 stars scanned so far, but all were written off when they failed to repeat.

The Berkeley effort, led by astronomer Dan Werthimer, takes a similar approach. Early next year, they will connect a laser detector to a 30-inch automated telescope at Berkeley's Leuschner Observatory that will observe 2,500 nearby stars. Meanwhile, Berkeley astronomer Geoff Marcy, codiscoverer of many planets outside the Solar System, is looking for laser signals among the data from his planet search.

Previous searches have almost all used radioastronomy. But according to Horowitz, recent technical advances have made optical SETI (search for extraterrestrial intelligence) a viable approach that is rapidly catching up with radio SETI. Whereas radio waves can better penetrate an atmosphere, lasers carry information more efficiently, and are not dispersed by the

608

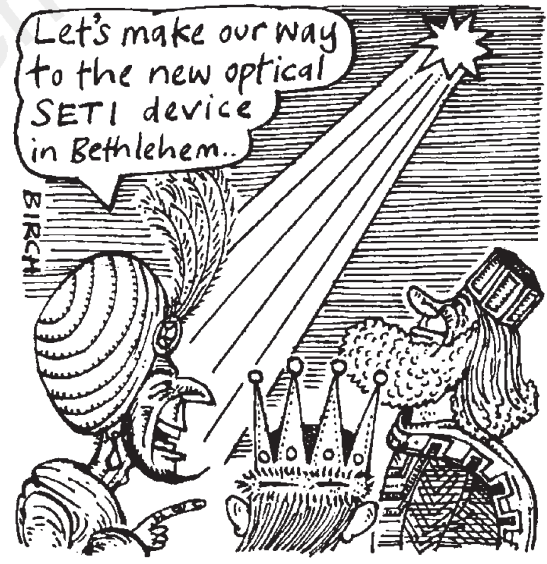

interstellar media to the same extent as radio waves.

Interference - a growing problem for radioastronomers on Earth because of the proliferation of cellular phones and communications satellites - is not a problem in optical wavelengths. With laser power output doubling every two years, Horowitz says, "we can now send out a pulse across the Galaxy more than a 1,000 times brighter than the Sun".

He believes a 'pulsed optical beacon' from a more advanced civilization could outshine its parent star by a factor of a million. These fast-talking extraterrestrials could signal to 1,000 stars in about a minute.

One of the biggest challenges currently facing SETI enthusiasts is deciding where in the heavens - and in the electromagnetic spectrum - to look. "If I were transmitting laser pulses, I'd do it in near-infrared rather than optical," Horowitz says, admitting that it's hard to know how to optimize the search. "Maybe they're sending 'zeta waves' and we haven't discovered them yet."

Even after searching for 20 years with no significant results, Horowitz is still optimistic. "We're the first generation that could realistically establish contact with another civilization, which would be the greatest event of all time, and almost no one is trying," he says.

"Earthlings are just learning how to do this," adds Werthimer. "The Universe could be filled with radio or laser signals shooting around, and we wouldn't have a clue." Searching for alien life is like looking for a needle in a haystack, he adds, but right now "Earthlings are just exploring the corners of the haystack. Eventually we will be able to search a large chunk of the haystack."

The new optical searches were stimulated partly by a workshop held last year by the California-based SETI Institute to design searches for the next two decades. "Most of what they are planning to do is more radio searches that cover larger chunks of the spectrum," says Horowitz. "That makes sense, but it doesn't mean we can't try something else too."

Horowitz was also inspired by a talk given in 1995 by Charles Townes, who won the Nobel prize for his work on lasers and masers, promoting the idea of optical SETI. "I was blown away thinking: 'How did I miss this?" he says. 